\title{
Fuzzy Methods and Approximate Reasoning in Geographical Information Systems
}

\author{
Ferdinando Di Martino, ${ }^{1}$ Irina Perfilieva, ${ }^{2}$ Salvatore Sessa, ${ }^{1}$ and Sabrina Senatore ${ }^{3}$ \\ ${ }^{1}$ Dipartimento di Architettura, Universitá degli Studi di Napoli Federico II, Via Toledo 402, 80134 Napoli, Italy \\ ${ }^{2}$ Centre of Excellence IT4Innovations, Institute for Research and Applications of Fuzzy Modelling, \\ University of Ostrava, 30. dubna 22, 70103 Ostrava, Czech Republic \\ ${ }^{3}$ Dipartimento di Informatica, Universitá degli Studi di Salerno, Via Ponte don Melillo, Fisciano, 80084 Salerno, Italy
}

Correspondence should be addressed to Ferdinando Di Martino; fdimarti@unina.it

Received 2 February 2014; Accepted 2 February 2014; Published 12 March 2014

Copyright (C) 2014 Ferdinando Di Martino et al. This is an open access article distributed under the Creative Commons Attribution License, which permits unrestricted use, distribution, and reproduction in any medium, provided the original work is properly cited.

This issue has been dedicated to the usage of fuzzy logic in the context of geographical information systems (GIS) and received the following papers whose contents are described below.

(i) In the paper of A. Hofmann et al., the authors use a GIS tool which is useful to study the influence of geographic and climatic factors on the terrain passability of armed forces and the integrated rescue system.

(ii) In the paper of F. Di Martino and S. Sessa, the authors propose the usage of the well-known extended GustafsonKessel clustering method, encapsulated in a GIS tool, for detecting hotspots in spatial analysis. The data consist of georeferenced patterns corresponding to positions of Taliban's attacks against civilians and soldiers in Afghanistan that happened during the period from 2004 to 2010: the formation through time of new hotspots is observed as well.

(iii) In the paper of M. Burda et al., an application of the so-called fuzzy GUHA method is presented for good peak prediction which was used in order to mine for fuzzy association rules expressed in natural language. The provided data was firstly extended by a creation of artificial variables describing various features of the data. The resulting variables were later on translated into fuzzy GUHA tables with help of evaluative linguistic expressions in order to mine for associations. The found associations were interpreted as fuzzy IF-THEN rules and used jointly with the perception-based logical deduction inference method to predict expected time shift of low rate peaks forecasted by the given physical model.

(iv) In another paper of F. Di Martino and S. Sessa, a fuzzy process for evaluating the reliability of a spatial database is defined: the area of study is partitioned in iso-reliable zones, defined as homogeneous zone in terms of data quality and environmental characteristics. This spatial database includes thematic datasets which in turn includes a set of layers. We estimate the reliability of each thematic dataset and therefore the overall reliability of the spatial database. This method is tested on the spatial dataset of the town of Cava de Tirreni (Italy) by means of a suitable GIS.

(v) In the paper of F. Di Martino et al., an application of the extended fuzzy C-means algorithm for detecting spatial areas with high concentrations of events, tested to study their temporal evolution, is proposed as well. This algorithm is implemented in a GIS tool. The data consist of georeferenced patterns corresponding to the residence of patients in the district of Naples (Italy) to whom a surgical intervention was carried out to the oto-laryngopharyngeal apparatus during the years from 2008 to 2012.

This special issue presents some noteworthy applications of the spatial analysis realized via GIS. Other applications should be desiderable in the sterminated world of GIS. We are aware that the topics do not easily meet desiderata of fuzzy authors; however we are at the beginning of a theory which is very promising from an applicational point of view, mainly in the spatiotemporal evolution of events which are either difficult to evaluate in the future or "fuzzy" for their same nature.

Ferdinando Di Martino Irina Perfilieva Salvatore Sessa Sabrina Senatore 

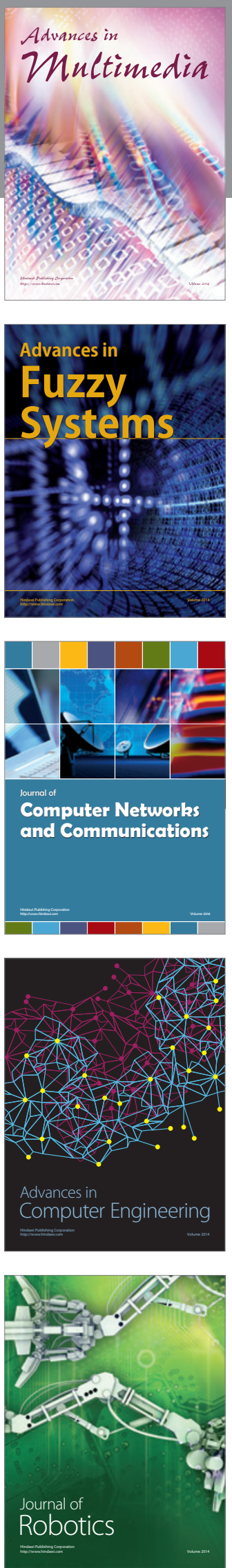

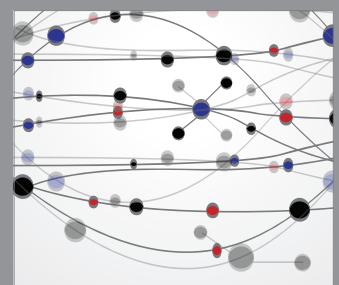

The Scientific World Journal
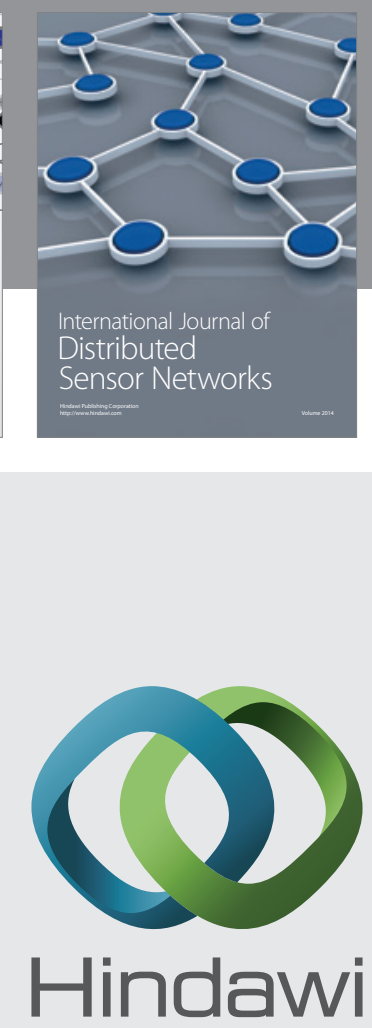

Submit your manuscripts at

http://www.hindawi.com
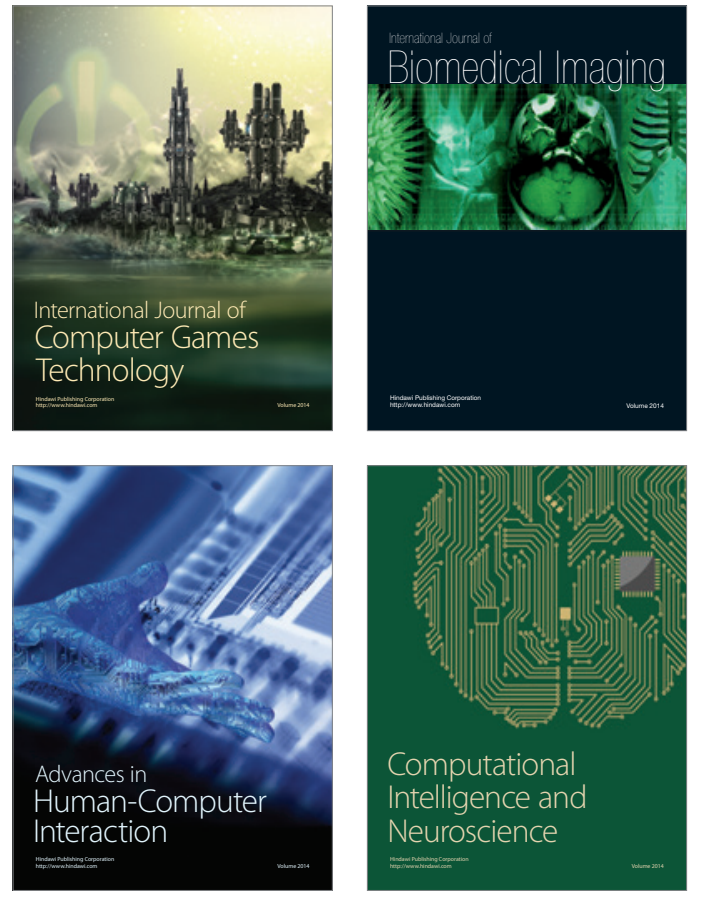
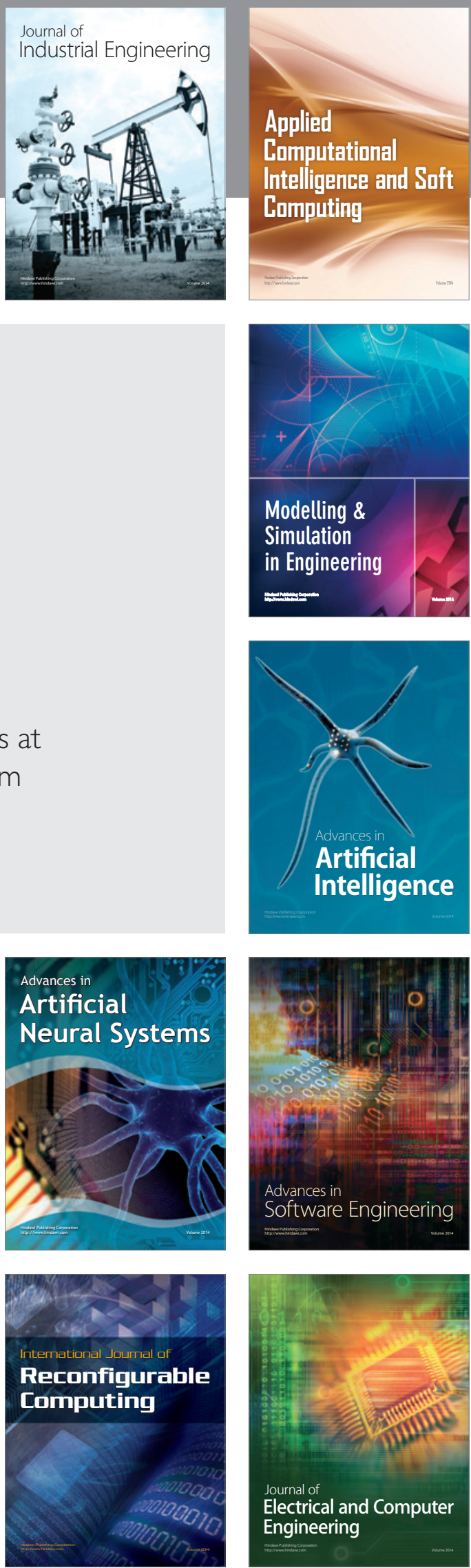